\title{
Modelo de Fatores para Commodities e Cenários de Preços no Curto Prazo: o caso da soja
}

\author{
Fernando Antonio Lucena Aiube ${ }^{1}$ \\ Bruna Carolina Fiúza Ferreira ${ }^{2}$ \\ Ariel Levy ${ }^{3}$
}

\section{Resumo}

Este artigo analisa os cenários futuros de curto prazo para o preço da soja à vista. A abordagem é feita modelando os fatores não observáveis por processos estocásticos e inova ao incluir o componente sazonal. As variáveis de observação são os preços futuros (estrutura a termo de contratos futuros). Procede-se à estimação do modelo pelo filtro de Kalman. Os resultados mostram um cenário de curto prazo mais favorável a produtores e exportadores. Por outro lado, consumidores e importadores têm indicação de medidas de proteção necessárias para suas posições. A análise pode ser estendida a outras commodities dependentes da sazonalidade. Da mesma forma, o modelo pode ser empregado no apreçamento de derivativos, em que o ativo subjacente é o preço futuro.

\section{Palavras-Chave}

Modelos em commodities. Contratos futuros da soja. Cenários de preços.

\begin{abstract}
This paper analyzes the short-term scenarios for soybeans spot prices. We include a seasonal component while modeling the non-observable variables through stochastic processes. The model was estimated using the Kalman filter over observable prices of future contracts term structure. The results show a more favorable scenario for producers and exporters. However, consumers and importers are indicated to hedge their positions. The analysis is extensible to other commodities dependent on seasonality. Likewise, the model can be used to price derivatives, where the future price is the underline asset.

- Agradecemos os comentários de dois pareceristas anônimos. Erros e omissões são de nossa responsabilidade.

1 Professor - Faculdade de Ciências Econômicas - Universidade do Estado do Rio de Janeiro Endereço: Rua São Francisco Xavier, 524 - Maracanã - Rio de Janeiro/RJ - Brasil - CEP: 20550-013. Email: faiube@gmail.com - ORCiD: https://orcid.org/0000-0003-3627-3484.

${ }^{2}$ Mestre - Programa de Pós-Graduação em Ciências Econômicas - Universidade do Estado do Rio de Janeiro - End.: Rua São Francisco Xavier, 524 - Maracanã - Rio de Janeiro/RJ - Brasil - CEP: 20550-013. Email: brunacffiuza@gmail.com - ORCiD: https://orcid.org/0000-0003-1220-9875.

${ }^{3}$ Professor - Faculdade de Administração e Ciências Contábeis - Universidade Federal Fluminense. Endereço: Rua Mário Santos Braga - Campus Valonguinho - Niterói/RJ - Brasil - CEP:24020-140. Email: alevy@id.uff.br - ORCiD: https://orcid.org/0000-0003-3557-1201.

Recebido: 31/10/2017. Aceite: 22/05/2019.

Editor Responsável: Dante Mendes Aldrighi
\end{abstract}

(c) (i) (\$) Esta obra está licenciada com uma Licença Creative Commons Atribuição-Não Comercial 4.0 Internacional. 


\section{Keywords}

Commodity models. Soybean futures. Price scenarios.

\section{Classificação JEL}

G13. C02. Q14.

\section{Introdução}

O objetivo deste artigo é analisar cenários de curto prazo para o preço da soja através de modelos que descrevem as variáveis relevantes por processos estocásticos. Como se trata de uma commodity agrícola, incluímos o componente sazonal na formulação do modelo e apresentamos a sua derivação de forma analítica.

A soja é uma commodity de grande relevância para a pauta de exportações do Brasil. Segundo o MDIC (Ministério da Indústria, Comércio Exterior e Serviços (2017)), a soja foi o produto mais exportado pelo Brasil em 2017, cujo valor arrecadado com a exportação da commodity foi de US\$ 25,71 bilhões, o que representa $11,81 \%$ do total das exportações brasileiras daquele ano. A magnitude da participação do Brasil no mercado mundial de soja deixa os agentes produtores fortemente expostos à demanda do mercado externo e às oscilações de preços.

A sazonalidade tem impacto relevante no preço de várias commodities, principalmente na análise de curto prazo, objetivo deste estudo. A primeira contribuição deste trabalho decorre da inclusão do componente sazonal no modelo de Gibson e Schwartz (1990). Este modelo, conforme observamos até o momento, não havia sido considerado com o componente sazonal. A segunda contribuição advém da derivação da equação do preço futuro a partir da solução do sistema de equações formado pelas variáveis estocásticas que definem os dois fatores. Os artigos originais de Gibson e Schwartz (1990) e de Schwartz (1997) apresentam a equação do preço futuro como solução de uma equação diferencial parcial (EDP). Por vezes a solução de uma EDP aumenta a complexidade do problema. A terceira contribuição decorre da análise de cenários de curto prazo para o preço da soja. A relevância desta análise para os agentes produtores está relacionada a momentos de grande oscilação de preços, como a que ocorreu no segundo semestre de 2014, quando os preços da soja tiveram uma queda abrupta. 
Neste caso, os produtores ficaram extremamente expostos a prejuízos. A análise de curto prazo sinaliza a necessidade de fazer proteção (hedge) para o consumidor/importador em seus negócios, buscando mitigar perdas futuras. Esta proteção normalmente envolve custos. Entretanto, caso seja sinalizado um cenário de recuperação de preços, tais custos de proteção poderão ser evitados. A análise dos cenários de preços contribui para além dos produtores, sendo útil também para os demais agentes do setor, gestores de risco nos mercados financeiros e órgãos governamentais interessados no planejamento e fomento da soja ou ainda no incremento de exportações de outras commodities.

A literatura em finanças considera, via de regra, que o preço à vista de commodities são variáveis não observáveis. De fato, o preço à vista, resultante de uma transação no mercado físico, não fica definido de imediato. O preço de fechamento ou liquidação (setllement price) de uma transação no mercado físico ocorre quando se dá o recebimento do produto pelo comprador. Por exemplo, se uma transação ocorre na data $t$, a commodity deve ser transportada do seu ponto de origem ao destino. A operação será liquidada em data posterior, em $t+\Delta t$. Portanto, o valor de liquidação estará associado à data $t+\Delta t$, quando se tem a entrega do produto. Mais ainda, o valor final da transação não é uma variável prontamente observável pelo mercado, somente é do conhecimento dos envolvidos na negociação do produto. Os valores dos preços das negociações são divulgados periodicamente por agências especializadas que coletam as informações junto aos agentes que negociam a commodity. Por outro lado, o preço futuro da commodity, negociado em bolsas de valores, é conhecido diariamente pelos canais de informação do mercado. Além de ser amplamente transparente, o preço futuro é formado competitivamente através de inúmeras transações diárias nas bolsas de mercados futuros. Em geral, o preço de uma commodity divulgado corriqueiramente pela imprensa é o preço do primeiro contrato futuro negociado nas bolsas de valores. Assim ocorre com o petróleo, a commodity mais relevante para a economia mundial, e com todas as demais que são negociadas em mercados futuros.

Os agentes de mercado têm grande interesse nas informações relacionadas ao preço à vista de uma commodity. Entretanto, trata-se de uma variável não observável, sendo comum que ela seja estimada. A literatura de finanças utiliza o filtro de Kalman para a estimação do preço à vista das commodities a partir das informações disponíveis do preço futuro das mesmas (variável observável). 
Este artigo tem por objetivo definir cenários para o preço da soja à vista usando tais procedimentos. Para tanto, tomamos as séries históricas de diferentes contratos futuros da soja negociados na CBOT (Chicago Board of Trade) e estimamos o preço à vista usando o filtro de Kalman. Foi utilizado o modelo de dois fatores de Gibson e Schwartz (1990). Os autores aplicam o modelo aos preços do petróleo onde não há necessidade de inclusão do componente sazonal. Neste trabalho, realizamos a derivação do modelo com a inclusão deste componente citado. Simultaneamente ao preço à vista, estimamos todos os hiperparâmetros do modelo. Após a estimação do modelo, são computadas a média e a variância do preço à vista sob a medida real de probabilidade. A partir do conhecimento da distribuição, baseado nesta medida, pode-se definir as probabilidades para diferentes cenários do preço à vista. Assim, de posse das informações disponíveis até a data $t$, calculamos os cenários de preços para a data $t+\Delta t$. Em outras palavras, computamos a probabilidade condicional $\operatorname{Prob}\left(S_{t+\Delta t}>S_{d} \mid \mathcal{F}_{t}\right)$, em que $\mathcal{F}_{t}$ é o conjunto de informações disponíveis até a data $t$ e $S_{d}$ é um valor do preço à vista de interesse dos agentes.

Este artigo foi organizado da seguinte forma: (i) a seção 2 apresenta uma revisão da literatura para os modelos de commodities com ênfase nos modelos de fatores estocásticos; (ii) a seção 3 apresenta o modelo utilizado; (iii) a seção 4 apresenta os dados utilizados; (iv) a seção 5 apresenta os resultados da estimação e simulações; e (v) a seção 6 contém as conclusões do estudo.

\section{Revisão da Literatura}

A literatura sobre previsão de preços para commodities é vasta. O grande esforço se concentra na área de energia, com destaque para os preços do petróleo. Há várias limitações nas metodologias empregadas nos diversos tipos de estudo. Pelo lado econométrico, usa-se frequentemente uma abordagem através do vetor autorregressivo (VAR) e vetor autorregressivo estruturado (SVAR). Quando há presença de variáveis exógenas em tais modelos, a previsão da variável dependente é função da previsão das variáveis exógenas. Citamos nesta direção os artigos de Baumeister e Kilian (2016) e Kilian (2014), dentre outros. Ainda com ênfase no petróleo, os estudos de Alquist et al. (2013) e Hamilton e Wu (2014) tratam do uso de diferentes 
abordagens presentes na literatura. Os métodos econométricos, em que se pressupõe que os preços passados carregam todas as informações relativas aos diversos fatores que causam suas alterações, também possuem algumas limitações. Novos choques que não ocorreram no passado não são capturados em tal abordagem. Não existe um consenso na literatura sobre qual o procedimento metodológico seja mais acurado. Neste estudo, seguimos a direção da modelagem dos preços por processos estocásticos. Neste caso, a dificuldade está na estimação de tais modelos. No entanto, algumas hipóteses simplificadoras, já bem estabelecidas na literatura, permitem a estimação adequada e são muito usadas no âmbito do apreçamento de derivativos em finanças. Uma síntese do uso de processos estocásticos na modelagem de preços em finanças pode ser vista em Shaffiee e Topal (2010), em que os autores fazem uma aplicação aos preços de longo prazo de combustíveis fósseis.

A literatura dos modelos de preços de commodities, elaborada a partir de fatores estocásticos, também é abundante. Apresentamos a seguir uma breve revisão da literatura dos modelos de fatores.

Os modelos de fatores aplicados a commodities iniciaram com o artigo de Brennan e Schwartz (1985). Os autores modelam o preço futuro da commodity a partir de um único fator estocástico, o preço à vista. Adotaram para a dinâmica do preço à vista o processo geométrico Browniano. O modelo foi aplicado no gerenciamento da operação (abertura, fechamento e abandono definitivo) de uma mina de cobre.

Em seguida, Gibson e Schwartz (1990) modelaram o preço da commodity com dois fatores estocásticos: o preço à vista e o retorno de conveniência (convenience yield). O preço à vista segue um processo geométrico Browniano. Nesta dinâmica está presente o retorno de conveniência. Este, por sua vez, segue um processo de Ornstein-Uhlenbeck. O modelo foi aplicado aos preços do petróleo. A derivação do modelo foi feita a partir da solução de uma EDP de segunda ordem.

Schwartz (1997) analisa o comportamento estocástico dos preços das commodities através de três modelos. O modelo de um fator contém apenas o preço à vista como fator estocástico. O modelo de dois fatores contém o preço à vista e o retorno de conveniência e é essencialmente o modelo definido em Gibson e Schwartz (1990). O modelo de três fatores inclui adicionalmente ao anterior a taxa de juros. Os modelos foram aplicados às 
commodities petróleo, cobre e ouro. A estimação nos três casos foi realizada com o filtro de Kalman. Inicialmente, coloca-se o modelo na forma espaço de estado. Os preços futuros constituem as variáveis de observação enquanto o preço à vista e o retorno de conveniência são as variáveis de estado (ou latentes, ou ainda de transição). $\mathrm{O}$ autor conclui pelo melhor desempenho do modelo de dois fatores no ajuste tanto da estrutura a termo de preços quanto na estrutura a termo da volatilidade.

Schwartz e Smith (2000) também apresentam um modelo de dois fatores em que o preço à vista é decomposto em um fator de curto prazo, cuja dinâmica estocástica segue um processo de Ornstein-Uhlenbeck e um fator de longo prazo, cuja dinâmica é um processo geométrico Browniano. Este modelo de dois fatores é aplicado aos preços do petróleo. Os autores usam o filtro de Kalman na estimação do modelo.

Nos artigos de Schwartz (1997) e Schwartz e Smith (2000), fica bem caracterizado que a inclusão de um fator estocástico, que representa o retorno de conveniência, contribui para uma melhor aderência aos dados empíricos observados.

Pilipovic (1998) apresenta um modelo de dois fatores em que o primeiro fator, preço à vista, evolui com a dinâmica de um processo de reversão. $\mathrm{O}$ segundo fator, o preço de longo prazo, possui a dinâmica de um processo geométrico Browniano. $\mathrm{O}$ autor apresenta o desenvolvimento do modelo do preço futuro por uma aproximação analítica.

Outros autores fazem uso do modelo básico de dois fatores de Schwartz e Smith (2000) para análise de comportamento de diferentes commodities. Dentre outros citamos: (i) Manoliu e Tompaidis (2002), que usaram este modelo para analisar o comportamento do gás natural; (ii) Lucia e Schwartz (2001), que aplicaram o modelo aos preços da eletricidade; e Sørensen (2002), que analisa o comportamento sazonal das commodities agrícolas (milho, soja e trigo).

Geman e Nguyen (2005), por sua vez, analisam, através de modelos de dois e três fatores, o comportamento da volatilidade com o inverso dos estoques (o inverso dos estoques foi definido como sendo a escassez do produto). Esses autores concluem que a volatilidade é uma função crescente da escassez e que a introdução da variável escassez no modelo de fatores melhora o ajuste comparativamente ao modelo de dois fatores. A estimação dos modelos foi feita com o filtro de Kalman. 
Cortazar e Naranjo (2006) propuseram um modelo com $N$ - fatores na investigação dos preços futuros do petróleo. A estimação também foi baseada no filtro de Kalman. Eles concluem que três fatores são suficientes para explicar o comportamento da estrutura a termo dos preços e que o uso de quatro fatores explica adequadamente a estrutura a termo da volatilidade.

Hikspoors e Jaimungal (2007) usam o modelo de dois fatores para o apreçamento de opções sobre o diferencial (spread) entre preços futuros. Usam o processo de reversão para o preço à vista e posteriormente fazem a inclusão de saltos nos valores dos preços. Eles obtêm, desta forma, uma solução fechada para o preço das opções com aplicações para o mercado de energia.

Do ponto de vista empírico, os trabalhos de Bessembinder et al. (1995) e Pindyck (1999) apresentam as evidências do comportamento de reversão para os preços das commodities. Postali e Picchetti (2006) analisam o comportamento dos preços do petróleo e argumentam que o processo geométrico Browniano tem uma boa aderência aos dados empíricos. A rejeição a tal processo ocorre somente com longas séries face à baixa velocidade de reversão. Por outro lado, a rejeição poderá ocorrer quando são omitidas as quebras estruturais.

Os modelos de preços para commodities mencionados até o momento possuem condições adequadas para a estimação dos preços. Trata-se de modelos Gaussianos e afins. Nestas circunstâncias, o filtro de Kalman é a metodologia adequada para esta estimação. As referências clássicas sobre o uso do filtro de Kalman na econometria são os textos de Harvey (1989), Hamilton (1994) e Durbin e Koopman (2002). Em finanças, além dos já citados acima, podemos acrescentar Babbs e Nowman (1999), que estimam o modelo de Vaciseck para taxa de juros.

Os modelos Gaussianos foram estendidos para abrigar mais adequadamente o comportamento empírico dos preços. Por exemplo, a inclusão de saltos torna o modelo não Gaussiano. Neste caso, o filtro de Kalman não é aplicado em sua forma canônica. Villaplana (2003), numa aplicação para os preços da energia elétrica, utiliza o modelo de dois fatores de Schwartz e Smith, e inclui a componente de saltos no fator de curto prazo. Aiube et al. (2008) seguem esta modelagem e utilizam o filtro de partículas para a estimação. Os autores fazem uma aplicação aos preços futuros do petróleo e concluem que o componente de saltos melhora a aderência aos dados empíricos. Javaheri et al. (2003) apresentam o modelo de volatilidade estocástica fazendo uso do filtro de partículas para a estimação. A inclusão 
da volatilidade como uma variável estocástica faz com que o modelo perca as propriedades Gaussianas.

A abordagem deste estudo considera a condição de Gaussianidade para as dinâmicas dos fatores estocásticos. A equação do preço futuro foi obtida pela solução analítica do sistema de equações estocásticas dos dois fatores. O resultado mostra que o preço futuro é uma função afim de tais fatores estocásticos. Portanto, a estimação é realizada com o uso do filtro de Kalman. Posteriormente, faz-se uso da lognormalidade do modelo para definição de cenários dos preços à vista.

\section{O Modelo de Dois Fatores}

Este estudo usa o modelo de dois fatores apresentado no artigo de Gibson e Schwartz (1990). Os dois fatores estocásticos são o preço à vista e o retorno de conveniência. O conceito do retorno de conveniência em commodities foi introduzido por Kaldor (1939) como sendo o benefício que possui o detentor da commodity física e que não é devido àquele que é proprietário de um contrato futuro. Trata-se de uma variável bem estabelecida dentro da literatura de commodities. O retorno de conveniência reflete as variações de curto prazo descritas pelos preços, tais como abundância e escassez da commodity. Quando os estoques estão elevados, o retorno de conveniência é baixo e os preços futuros também. Quando os estoques caem, o retorno de conveniência é alto. $\mathrm{O}$ retorno de conveniência é uma proxy para o nível dos estoques. Para uma commodity global, como a soja, o nível dos estoques é de difícil mensuração. Isto torna o retorno de conveniência uma variável útil para descrever o nível dos estoques que notadamente é refletido nos preços. Geman (2005) ressalta que o retorno de conveniência é muito apropriado para descrever o comportamento das diferentes formas de estrutura a termo dos preços futuros. Os conceitos sobre os determinantes do retorno de conveniência podem ser encontrados em Prokopczuk e Wu (2013).

Outros modelos de fatores poderiam ser aplicados a este estudo, no entanto, a maioria dos estudos considera o uso de fatores genéricos. Alguns utilizam a interpretação de curto e longo prazos. Em outros casos, os fatores ficam estabelecidos sem uma interpretação precisa, o que dificulta o seu entendimento. Em Gibson e Schwartz (1990), os fatores estocásticos 
(preço à vista e retorno de conveniência) são diretamente modelados e constituem conceitos bem fundamentados dentro da literatura.

Nos artigos originais de Gibson e Schwartz (1990) e Schwartz (1997), o modelo não contém o componente sazonal. Além disso, a equação para o preço futuro é apresentada como a solução de uma EDP, como já foi aqui explicado anteriormente, o que envolve uma complexa solução.

Neste artigo, ampliamos o modelo pela inclusão do componente sazonal. Simultaneamente, apresentamos o preço futuro como solução de um sistema de equações diferenciais estocásticas que descreve as dinâmicas dos dois fatores. Desta forma, usamos a seguir esta solução analítica para a estimação do modelo. De posse dos parâmetros, podemos definir cenários para o preço à vista sob a condição de lognormalidade do mesmo.

Podemos considerar $(\Omega, \mathbb{F}, \mathbb{P})$ como o espaço de probabilidade que descreve a incerteza na economia. Onde $\Omega$ é o espaço de eventos, $\mathbb{F}=\{\mathcal{F}\}_{0 \leq t \leq T}$ representa a filtração natural dos Brownianos associados às incertezas dos dois processos e $\mathbb{P}$ a medida de probabilidade real ou histórica. Por suposição, consideramos que o mercado é completo e deste fato decorre a existência e unicidade da medida martingal equivalente (MME), e escrevemos $\mathbb{Q} \sim \mathbb{P}$.

O modelo de dois fatores estocásticos é descrito por duas equações diferenciais estocásticas para as variáveis (ou fatores): preço à vista $S_{t}$ e retorno de conveniência $\delta_{t}$.

O primeiro fator estocástico $S_{t}$ segue um processo geométrico Browniano. O segundo fator estocástico $\delta_{t}$ evolui como um processo de OrnsteinUhlenbeck. Ambos os processos estão definidos abaixo sob a medida real de probabilidade $\mathbb{P}$. Seja então $t \epsilon[0, T]$ em que $T$ representa o vencimento do contrato futuro.

Para incorporar a sazonalidade definimos:

$$
S_{t}=e^{f_{t}+X_{t}}
$$

em que $f_{t}$ é uma função determinística de senos e cossenos e $X_{t}$ tem a dinâmica conforme a equação descrita abaixo:

$$
d X_{t}=\left(\mu-\delta_{t}-\frac{1}{2} \sigma_{1}^{2}\right) d t+\sigma_{1} d B_{1, t}
$$


em que $\mu$ é o retorno total esperado do proprietário da commodity; $\sigma_{1}>0$ é a volatilidade de $X_{t} ; d B_{1, t}$ é o incremento do processo Browniano padrão para $X_{t}$ e $\delta_{t}$ é o retorno de conveniência instantâneo. A sua dinâmica é dada pelo processo de Ornstein-Uhlenbeck conforme a equação abaixo:

$$
d \delta_{t}=\kappa\left(\alpha-\delta_{t}\right) d t+\sigma_{2} d B_{2, t}
$$

em que $\kappa>0$ é a velocidade de reversão; $\alpha$ é a média de longo prazo de $\delta_{t}$; $\sigma_{2}>0$ a sua volatilidade; e $d B_{2, t}$ é o incremento do processo Browniano padrão para $\delta_{t}$. Além disso, os incrementos dos processos Brownianos padrão $d B_{1, t}$ e $d B_{2, t}$ são correlacionados por $\rho$, de tal forma que $d B_{1, t} d B_{2, t}=\rho d t$.

Neste modelo, o preço futuro será função de dois fatores de risco. Assim, escrevemos que o preço na data $t$, de um contrato futuro com vencimento em $T$, é $F_{t, T}\left(S_{t}, \delta_{t}\right)$. Em outras palavras, o preço futuro pode ser descrito como a função das duas variáveis estocásticas. A proposição a seguir define a equação do preço futuro.

Proposição: Considere o vencimento de um contrato futuro na data $T$ e o instante atual como a data zero. Sendo $F_{0, T}=E^{\mathbb{Q}}\left(S_{T} \mid \mathcal{F}_{0}\right)$ e usando as Equações (2) e (3) acima, demonstra-se que:

$$
\ln F_{0, T}=\ln S_{0}-\delta_{0} \frac{1-e^{-\kappa T}}{\kappa}+A(T)
$$

em que

$$
\begin{aligned}
A(T)=f_{T}+ & \left(r-\widehat{\alpha}+\frac{\sigma_{2}^{2}}{2 \kappa}-\frac{\sigma_{1} \sigma_{2} \rho}{\kappa}\right) \mathrm{T}+\left(\kappa \widehat{\alpha}+\sigma_{1} \sigma_{2} \rho-\frac{\sigma_{2}^{2}}{k}\right) \frac{1-e^{-\kappa T}}{\kappa^{2}} \\
& +\frac{\sigma_{2}^{2}}{4 \kappa^{3}}\left(1-e^{-2 \kappa T}\right)-f_{0}
\end{aligned}
$$

e ainda $\hat{\alpha}=\alpha-\frac{\lambda}{\kappa}$, em que $\lambda$ é o preço do risco de mercado do retorno de conveniência.

Prova: A demonstração encontra-se no Apêndice 1 .

Note-se que tanto em Gibson e Schwartz (1990) como em Schwartz (1997), o preço do contrato futuro $F_{t, T}$ é obtido a partir da solução de uma EDP. Neste artigo, a solução provém da solução do sistema de equa- 
ções diferenciais estocásticas que definem as dinâmicas das variáveis $S_{t}$ e $\delta_{t}$ sob a MME.

A função determinística, que representa a sazonalidade, pode ser definida de várias formas. Seguimos a formulação de Hikspoors e Jaimungal (2007) em que temos:

$$
f(t)=\sum_{i=1}^{n} \beta_{i} t+\alpha_{1 i} \operatorname{sen}(2 \pi t)+\alpha_{2 i} \cos (2 \pi t)
$$

em termos práticos, para a estimação, usamos apenas um termo para o somatório na função acima.

A etapa seguinte é a implementação da estimação do modelo. Sendo um modelo Gaussiano com uma estrutura afim, o procedimento canônico é a estimação pelo filtro de Kalman. Na linguagem do filtro de Kalman, a variável de observação é o preço futuro. As variáveis de estado ou transição são aquelas não observáveis, que no presente caso são $X_{t}$ e $\delta_{t}$. Desta forma, a equação de observação do modelo pode ser descrita por:

$$
\boldsymbol{F}_{t, T}=\widehat{\boldsymbol{F}}_{t, T}+\boldsymbol{\epsilon}_{t}
$$

em que $\boldsymbol{F}_{t, T}$ é um vetor coluna com o número de linhas igual ao número de contratos observados contendo os preços em cada instante de tempo $t$. Já $\widehat{\boldsymbol{F}}_{t, T}$ representa o exponencial da Equação (4), que define o modelo dos preços futuros, o que foi aqui deduzido e $\boldsymbol{\epsilon}_{t}$ é um vetor de erro em que cada elemento é normal e identicamente distribuído (NID). Assim escrevemos, $\epsilon_{i_{t}} \sim \operatorname{NID}\left(0, s_{\epsilon i}^{2}\right)$, com $i$ representando cada contrato futuro que compõe o vetor.

As equações com as dinâmicas das variáveis de estado estão descritas nas Equações (2) e (3) sob a medida real de probabilidade. Em suma, as Equações (2), (3), (5) e (6) definem o modelo a ser estimado. O Apêndice 2 apresenta os detalhes para a implementação da estimação do modelo, descrevendo-o na forma espaço de estado.

Uma vez realizada a estimação do modelo, podemos usar os parâmetros estimados para calcular a média e variância, na medida real $\mathbb{P}$, para a variável $X_{t}$.

Corolário: A variável $X_{t}$ tem distribuição normal com média e variância, sob a medida $\mathbb{P}$, dadas por: 


$$
\begin{gathered}
E^{\mathbb{P}}\left(X_{t} \mid \mathcal{F}_{0}\right)=X_{0}+\left(\mu-\frac{1}{2} \sigma_{1}^{2}\right) t-\alpha t+\frac{\alpha-\delta_{0}}{\kappa}\left(1-e^{-\kappa t}\right) \\
\operatorname{Var}^{\mathbb{P}}\left(X_{t} \mid \mathcal{F}_{0}\right)=\left(\sigma_{1}^{2}+\frac{\sigma_{2}^{2}}{\kappa^{2}}-\frac{2 \sigma_{1} \sigma_{2} \rho}{\kappa}\right) t-2 \frac{\sigma_{2}^{2}}{\kappa^{3}}\left(1-e^{-\kappa t}\right)+ \\
2 \frac{\sigma_{1} \sigma_{2} \rho}{\kappa^{2}}\left(1-e^{-\kappa t}\right)+\frac{\sigma_{2}^{2}}{2 \kappa^{3}}\left(1-e^{-2 \kappa t}\right)
\end{gathered}
$$

Prova: Seguindo a demonstração da Proposição no Apêndice 1, temos que: (i) a Equação (A6) define em $t=T$, em que $X_{T}$ tem distribuição normal (isto é uma consequência direta da definição de $X_{t}$ na Equação (2)); (ii) a Equação (A7) define a média sob a medida $\mathbb{P}$, e (iii) a Equação (A17) define a variância, que é a mesma sob ambas as medidas de probabilidade.

Portanto, esta variável tem distribuição normal em qualquer instante. Denominamos por $\bar{T}$ a data de interesse para avaliarmos o preço à vista. Da definição na Equação (1), temos que $S_{\bar{T}}=e^{f_{\bar{T}}+X_{\bar{T}}}$, e sabemos que o preço à vista tem distribuição lognormal. Assim, pode-se computar a probabilidade condicional do preço à vista $S_{\bar{T}}$ como sendo maior ou menor que um valor exógeno de interesse. Por exemplo, deseja-se investigar o cenário de que o preço à vista seja superior a US\$ 1.500 cents/bushel seis meses à frente. Desta forma, computamos a probabilidade $\operatorname{Prob}\left(S_{\bar{T}}>1500 \mid \mathcal{F}_{t}\right)$, em que $\bar{T}$ representa a data daqui a seis meses e $\mathcal{F}_{t}$ o conjunto de informações na data atual $t$ (em termos matemáticos $\mathcal{F}_{t}$ significa a sigma-álgebra em $t$ ).

\section{Dados}

Para a estimação do modelo, tomamos os preços dos contratos futuros negociados na bolsa CBOT com frequência semanal. O período observado como amostra abrange as informações dos preços futuros de 03/01/1997 a 14/04/2017, com vencimentos variados. Este período abrange os principais eventos recentes do movimento de preços tais como a financialização das commodities e a queda abrupta verificada no segundo semestre de 2014 . A série dos preços futuros foram denominadas por $F_{i}$, em que $i$ representa o número de meses futuros para o vencimento do contrato. Os preços são 
cotados em US\$ cents/bushel. A Tabela 1 contém as principais estatísticas das séries tidas como amostra.

A taxa livre de risco foi considerada como a média da taxa de juros no mercado americano durante o período referente à amostra coletada acima. O valor calculado é de aproximadamente $3 \%$ ao ano.

Tabela 1: Principais estatísticas das séries dos preços futuros

\begin{tabular}{|l|c|c|c|c|c|}
\hline & $\boldsymbol{F}_{\mathbf{1}}$ & $\boldsymbol{F}_{\mathbf{3}}$ & $\boldsymbol{F}_{\mathbf{5}}$ & $\boldsymbol{F}_{\mathbf{7}}$ & $\boldsymbol{F}_{\boldsymbol{8}}$ \\
\hline Média & 874.95 & 864.66 & 851.27 & 842.85 & 847.07 \\
\hline Desvio Padrão & 338.93 & 325.77 & 310.11 & 298.16 & 293.61 \\
\hline Mínimo & 414.5 & 410.25 & 422 & 435.25 & 440 \\
\hline Máximo & 1763.25 & 1757.5 & 1644.75 & 1616.5 & 1623.5 \\
\hline Assimetria & 0.49 & 0.48 & 0.45 & 0.43 & 0.4 \\
\hline Curtose & -0.91 & -0.94 & -1.04 & -1.08 & -1.11 \\
\hline
\end{tabular}

Nota: Os dados de curtose referem-se ao excesso de curtose para a série de preços.

A Figura 1 apresenta o gráfico do primeiro contrato futuro $F_{1}$. Alguns momentos do mercado chamam a atenção. Primeiro, os picos dos preços ocorrem antes e após a crise financeira em setembro de 2008. Segundo, após 2005, há uma tendência clara de aumento dos preços (trata-se da financialização). Terceiro, no segundo semestre de 2014, há uma forte queda nos preços. Tais movimentos são observados na maioria das séries de preços das commodities.

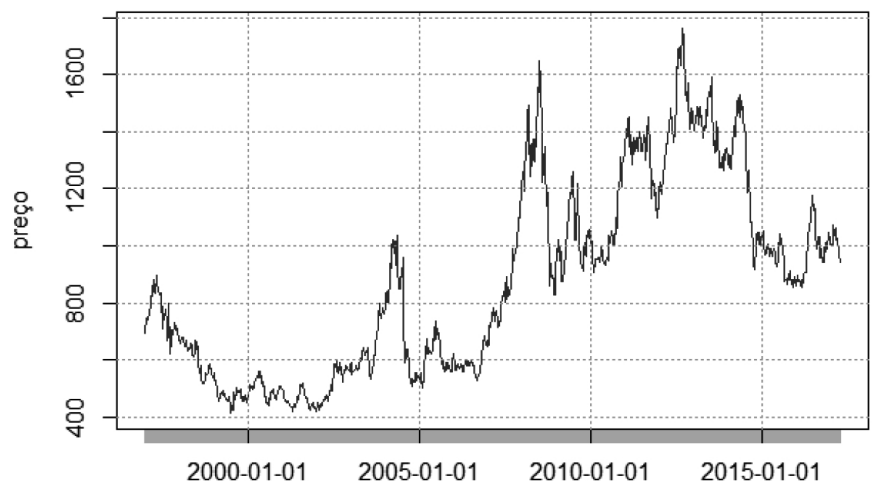

Figura 1 - Preço do primeiro contrato futuro em US\$ cents/bushel 


\section{Resultados}

A Tabela 2 apresenta os resultados da estimação. Nota-se que tanto a velocidade de reversão quanto as volatilidades são significantes. O mesmo pode ser observado sobre o componente sazonal relativo à parte trigonométrica. Conforme esperado, o estimador do parâmetro $\mu$ se apresenta como não significante. Para estimação mais acurada deste parâmetro, é necessário dispor de séries históricas mais longas devido à característica de sua distribuição assintótica. Este fato é bem descrito em Campbell et al. (1997). O parâmetro $\rho$ não significante reflete a baixa ortogonalidade contemporânea ente as duas variáveis de estado do modelo. O parâmetro $\lambda$, preço de risco de mercado, é não significante. Este resultado pode de fato ocorrer, significando que, para este conjunto de dados, o parâmetro pode ser tomado como aproximadamente zero. Resultado idêntico para o parâmetro $\lambda$ foi obtido por Schwartz (1997), que também usa o filtro de Kalman. Todos os parâmetros referentes à variância do termo de erro são altamente significantes.

Tabela 2 - Estimação dos hiperparâmetros do modelo

\begin{tabular}{|c|c|c|c|}
\hline Parâmetro & Valor estimado & Erro padrão & Estatística t \\
\hline $\boldsymbol{\mu}$ & 0,291 & 0,182 & 1,60 \\
\hline $\boldsymbol{\kappa}$ & 0,979 & 0,072 & 13,49 \\
\hline $\boldsymbol{\alpha}$ & 0,046 & 0,068 & 0,69 \\
\hline $\boldsymbol{\sigma}_{\boldsymbol{1}}$ & 0,797 & 0,025 & 31,06 \\
\hline $\boldsymbol{\sigma}_{2}$ & 0,031 & 0,002 & 12,08 \\
\hline $\boldsymbol{\rho}$ & 0,220 & 0,368 & 0,6 \\
\hline $\boldsymbol{\lambda}$ & 0,028 & 0,053 & 0,53 \\
\hline $\boldsymbol{s}_{\boldsymbol{\epsilon}}^{2}$ & $2 \times 10^{-4}$ & $1 \times 10^{-6}$ & 125,62 \\
\hline $\boldsymbol{s}_{\boldsymbol{\epsilon} \mathbf{2}}^{2}$ & $6 \times 10^{-4}$ & $6 \times 10^{-7}$ & 1049,62 \\
\hline $\boldsymbol{s}_{\boldsymbol{\epsilon} \mathbf{2}}^{2}$ & $7 \times 10^{-5}$ & $2 \times 10^{-6}$ & 25,04 \\
\hline $\boldsymbol{s}_{\boldsymbol{\epsilon} \mathbf{2}}^{2}$ & $1 \times 10^{-3}$ & $1 \times 10^{-6}$ & 929,67 \\
\hline $\boldsymbol{s}_{\boldsymbol{\epsilon} \boldsymbol{8}}^{2}$ & $8 \times 10^{-3}$ & $6 \times 10^{-6}$ & 1344,64 \\
\hline $\boldsymbol{\alpha}_{1}$ & 0,009 & 0,003 & 3,27 \\
\hline $\boldsymbol{\beta}_{\mathbf{1}}$ & 0,05 & 0,133 & 0,37 \\
\hline $\boldsymbol{\alpha}_{2}$ & 0,006 & 0,002 & 2,99 \\
\hline
\end{tabular}

A Figura 2 apresenta o logaritmo das duas variáveis de estado não observáveis estimadas pelo filtro de Kalman. 


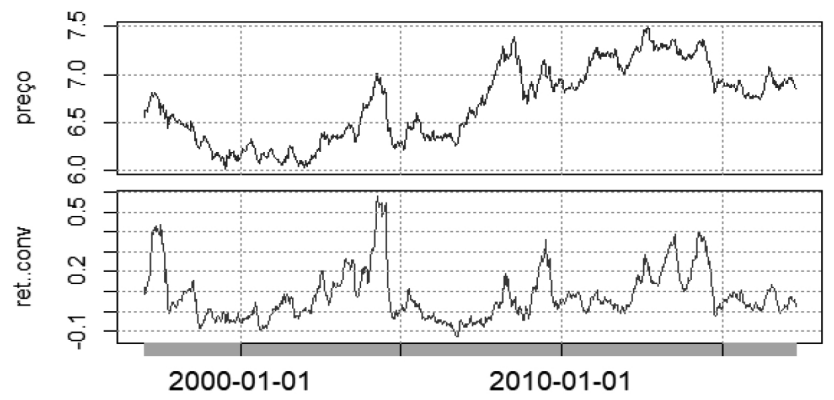

Figura 2 - Preço à vista no painel superior e retorno de conveniência no inferior, em logaritmo

Para o cálculo da média e variância da distribuição de $X_{t}$ usamos o valor inicial do preço do primeiro contrato futuro como proxy para o preço à vista, isto é $S_{0}=$ US\$ 996,4 cents/bushel referente a 11/05/2018 na CBOT. Computamos cenários de preços para 3, 6, 9 e 12 meses à frente da data acima.

A Tabela 3 resume os cenários analisados. Para a situação de baixa, investigamos a probabilidade do preço à vista ser inferior aos níveis de US\$ 800 cents/bushel e US\$ 600 cents/bushel. Nota-se que em um horizonte de um ano, estas probabilidades são de 39\% e 26\%, respectivamente. Fazendo o mesmo cálculo para a média de longo prazo de US\$ 875 cents/bushel (estimado como proxy para o preço à vista a partir do primeiro contrato futuro), observa-se que a probabilidade de um cenário inferior a este preço é de $43 \%$ no horizonte de um ano. Portanto, o cenário de preços maiores que a média de longo prazo é bastante favorável.

Tabela 3 - Probabilidades para os cenários de preços

\begin{tabular}{|l|c|c|c|c|}
\hline \multirow{2}{*}{ Cenários } & \multicolumn{4}{|c|}{ Períodos à frente } \\
\cline { 2 - 5 } & 3 meses & 6 meses & 9 meses & 12 meses \\
\hline $\operatorname{Prob}\left(S_{\bar{T}}<600 \mid S_{t}=996,4\right)$ & $9,6 \%$ & $18,54 \%$ & $23,38 \%$ & $25,85 \%$ \\
\hline $\operatorname{Prob}\left(S_{\bar{T}}<800 \mid S_{t}=996,4\right)$ & $27,97 \%$ & $34,98 \%$ & $37,85 \%$ & $38,72 \%$ \\
\hline $\operatorname{Prob}\left(S_{\bar{T}}<875 \mid S_{t}=996,4\right)$ & $36,07 \%$ & $41,07 \%$ & $42,87 \%$ & $43,03 \%$ \\
\hline $\operatorname{Prob}\left(S_{\bar{T}}>1300 \mid S_{t}=996,4\right)$ & $26,24 \%$ & $31,65 \%$ & $34,61 \%$ & $37,27 \%$ \\
\hline $\operatorname{Prob}\left(S_{\bar{T}}>1500 \mid S_{t}=996,4\right)$ & $15,98 \%$ & $23,18 \%$ & $27,8 \%$ & $30,67 \%$ \\
\hline $\operatorname{Prob}\left(S_{\bar{T}}>1763 \mid S_{t}=996,4\right)$ & $8,08 \%$ & $15,43 \%$ & $20,01 \%$ & $23,97 \%$ \\
\hline
\end{tabular}

Nota: Na tabela, representamos o conjunto de informações $\mathcal{F}_{t}$ por $S_{t}$. 
Para a situação de alta, analisamos cenários do preço à vista superar US\$ 1.300 cents/bushel e US\$ 1.500 cents/bushel. No horizonte de um ano, estas probabilidades atingem $37 \%$ e $31 \%$, respectivamente. A probabilidade de preços superiores ao máximo de US\$ 1.763 cents/bushel é de 24\%. Fica evidente que os cenários são promissores para os produtores e, consequentemente, para os países exportadores. Por outro lado, há uma sinalização de maiores gastos com a importação do produto para os países que demandam a soja. Pode-se também inferir que os produtos que utilizam a soja como insumo, como a ração animal, por exemplo, estão propensos a terem seus preços mais elevados, conforme o cenário simulado acima. A situação adversa de consumidores/importadores pode ser mitigada fazendo-se proteção (hedge) de sua exposição. Por exemplo, através de uma posição comprada (long) no mercado futuro.

\section{Conclusões}

Este artigo analisou os cenários de preços de curto prazo para os preços à vista da soja através do modelo de dois fatores de Gibson e Schwartz (1990). A componente sazonal foi introduzida neste modelo para tratar commodities que são dependentes de tais efeitos. Por outro lado, foi realizada a derivação do modelo do preço para contratos futuros da commodity sem a necessidade de se recorrer aos resultados advindos de soluções de EDP's. Trata-se de um modelo Gaussiano e afim. A estimação foi realizada a partir da observação da estrutura a termo dos preços futuros. Seguimos a forma canônica para tais casos usando o filtro de Kalman.

Os resultados advindos da simulação de cenários de preços à vista no curto prazo são favoráveis aos agentes produtores e/ou países exportadores. No curto prazo, os consumidores e/ou importadores têm uma sinalização de maiores gastos com a aquisição da soja. Os resultados deste estudo são úteis para os agentes mais expostos a riscos que podem tomar medidas para mitigá-los.

Este estudo tem várias aplicações e extensões. Por exemplo, o modelo pode ser aplicado a outras commodities que estão sujeitas aos efeitos sazonais, tais como àquelas do setor de energia (gás natural, derivados do petróleo e eletricidade) e às demais commodities relacionadas ao agronegócio. 
O estudo pode ser estendido para análise do preço à vista das commodities relacionadas à soja. O processamento da soja produz duas outras commodities: o farelo e o óleo de soja. Desta forma, o diferencial entre os preços dos produtos da soja (farelo e óleo) e o preço da soja bruta constitui a margem de ganho da indústria de processamento. Este diferencial (spread) de preços, que também está sujeito à sazonalidade, pode ser estimado a partir da modelagem desenvolvida neste artigo. Mais ainda, os modelos para os contratos de opções financeiras sobre o preço futuro, comumente negociados em bolsas de valores, fazem uso da modelagem do preço futuro e podem ser derivados a partir dos resultados aqui encontrados.

\section{Apêndice 1}

\section{Modelo de dois fatores de Gibson e Schwartz para os preços das com- modities}

Neste Apêndice, são apresentados os detalhes da solução analítica do conjunto de Equações diferencias estocásticas (2) e (3) que definem o modelo para o preço futuro: $F_{0, T}$.

Os pressupostos para a derivação descrita abaixo estão no corpo deste artigo. Inicialmente apresentamos o modelo sob a medida real de probabilidade.

Para incorporar a sazonalidade ao modelo definimos a variável $X_{t}$ de tal forma que:

$$
S_{t}=e^{f_{t}+X_{t}}
$$

em que $S_{t}$ é o preço à vista, $f(t)$ é uma função determinística de senos e cossenos, e $X_{t}$ tem a dinâmica conforme a descrita abaixo:

$$
d X_{t}=\left(\mu-\frac{1}{2} \sigma_{1}^{2}\right) d t-\delta_{t} d t+\sigma_{1} d B_{1, t}
$$

Na Equação (A2), a variável $\delta_{t}$ é o retorno de conveniência. Sua dinâmica segue o processo:

$$
d \delta_{t}=\kappa\left(\alpha-\delta_{t}\right) d t+\sigma_{2} d B_{2, t}
$$


As Equações (A1), (A2) e (A3) descrevem o modelo de dois fatores sob a medida real de probabilidade $\mathbb{P}$. A solução da Equação (A3) para $\delta_{t}$, sendo $t \in[0, T]$, é obtida integrando-se entre a data $t=0$ e $t$, em que se obtém:

$$
\delta_{t}=\alpha+\left(\delta_{0}-\alpha\right) e^{-\kappa t}+\sigma_{2} \int_{0}^{t} e^{\kappa(u-t)} d B_{2, u}
$$

Integrando a Equação (A2) de zero a $t$ temos:

$$
X_{t}=X_{0}+\left(\mu-\frac{1}{2} \sigma_{1}^{2}\right) t-\int_{0}^{t} \delta_{u} d u+\sigma_{1} \int_{0}^{t} d B_{1, u}
$$

Integrando a Equação (A4) e introduzindo o resultado obtido na Equação (A5), temos:

$$
\begin{aligned}
X_{t}=X_{0}+\left(\mu-\frac{1}{2} \sigma_{1}^{2}\right) t-\alpha t & +\frac{\alpha-\delta_{0}}{\kappa}\left(1-e^{-\kappa t}\right)-\frac{\sigma_{2}}{\kappa} \int_{0}^{t} d B_{2, u} \\
+ & \frac{\sigma_{2}}{\kappa} \int_{0}^{t} e^{\kappa(u-t)} d B_{2, u}+\sigma_{1} \int_{0}^{t} d B_{1, u}
\end{aligned}
$$

O valor esperado de $X_{t}$, sob a medida $\mathbb{P}$, condicionado às informações disponíveis em $t=0$ é:

$$
E^{\mathbb{P}}\left(X_{t} \mid \mathcal{F}_{0}\right)=X_{0}+\left(\mu-\frac{1}{2} \sigma_{1}^{2}\right) t-\alpha t+\frac{\alpha-\delta_{0}}{\kappa}\left(1-e^{-\kappa t}\right)
$$

Usando a isometria de Itô, podemos calcular a variância de $X_{t}$ sob a medida $\mathbb{P}$. Como a variância é a mesma em ambas as medidas, apresentamos este resultado ao tratarmos do modelo sob a medida $\mathbb{P}$.

Descrevemos, ainda, o modelo dado pelas Equações (A2) e (A3) sob a MME. Neste caso, a dinâmica de $X_{t}$ é obtida mudando o $\mu$ pela taxa livre de risco $r$, assim encontramos:

$$
d X_{t}=\left(r-\frac{1}{2} \sigma_{1}^{2}\right) d t-\delta_{t} d t+\sigma_{1} d \tilde{B}_{1, t}
$$

em que $d \tilde{B}_{i}, i=1,2$ representam os incrementos dos processos Brownianos sob a MME. A dinâmica do retorno de conveniência sob a MME é obtida pela introdução do preço de risco do mercado $\lambda$, considerado constante em $[0, T]$, por conseguinte, temos:

$$
d \delta_{t}=\left[\kappa\left(\alpha-\delta_{t}\right)-\lambda\right] d t+\sigma_{2} d \tilde{B}_{2, t}
$$


A Equação (A9) pode ser reescrita como:

$$
d \delta_{t}=\kappa\left(\hat{\alpha}-\delta_{t}\right) d t+\sigma_{2} d \tilde{B}_{2, t}
$$

em que $\hat{\alpha}=\alpha-\frac{\lambda}{\kappa}$ define a média de longo prazo do retorno de conveniência sob a MME. A solução da Equação (A10) é dada por:

$$
\delta_{t}=\hat{\alpha}+\left(\delta_{0}-\hat{\alpha}\right) e^{-\kappa t}+\sigma_{2} \int_{0}^{t} e^{\kappa(u-t)} d \tilde{B}_{2, u}
$$

Similarmente à Equação (A15), podemos observar:

$$
X_{t}=X_{0}+\left(r-\frac{1}{2} \sigma_{1}^{2}\right) t-\int_{0}^{t} \delta_{u} d u+\sigma_{1} \int_{0}^{t} d \tilde{B}_{1, u}
$$

Integrando a Equação (All) de zero a $t$ e introduzindo este resultado na Equação (A12), chegamos ao resultado para $X_{t}$ sob a MME, isto é:

$$
\begin{gathered}
X_{t}=X_{0}+\left(r-\frac{1}{2} \sigma_{1}^{2}\right) t-\hat{\alpha} t+\frac{\hat{\alpha}-\delta_{0}}{\kappa}\left(1-e^{-\kappa t}\right)-\frac{\sigma_{2}}{\kappa} \int_{0}^{t} d \tilde{B}_{2, u} \\
+\frac{\sigma_{2}}{\kappa} \int_{0}^{t} e^{\kappa(u-t)} d \tilde{B}_{2, u}+\sigma_{1} \int_{0}^{t} d \tilde{B}_{1, u}
\end{gathered}
$$

O valor esperado de $X_{t}$ sob a MME, condicionado às informações disponíveis na data $t=0$, é dado assim por:

$$
E^{\mathbb{Q}}\left(X_{t} \mid \mathcal{F}_{0}\right)=X_{0}+\left(r-\frac{1}{2} \sigma_{1}^{2}\right) t-\hat{\alpha} t+\frac{\widehat{\alpha}-\delta_{0}}{\kappa}\left(1-e^{-\kappa t}\right)
$$

Podemos reescrever a Equação (A13) de forma simplificada, em que as integrais estocásticas do segundo membro serão denominadas por $I_{1}, I_{2}$ e $I_{3}$, respectivamente. Usando o resultado da Equação (Al4) chegamos a:

$$
X_{t}=E^{\mathbb{Q}}\left(X_{t} \mid \mathcal{F}_{0}\right)-\frac{\sigma_{2}}{\kappa} I_{1}+\frac{\sigma_{2}}{\kappa} I_{2}+\sigma_{1} I_{3}
$$

A variância condicional de $X_{t}$ sob a MME, envolve o cálculo da variância de cada integral estocástica e as covariâncias entre as mesmas. Portanto, temos:

$$
\begin{array}{r}
\operatorname{Var}^{\mathbb{Q}}\left(X_{t} \mid \mathcal{F}_{0}\right)=\frac{\sigma_{2}^{\llcorner}}{\kappa^{2}} \operatorname{Var}^{\mathbb{Q}}\left(I_{1} \mid \mathcal{F}_{0}\right)+\frac{\sigma_{2}^{\llcorner}}{\kappa^{2}} \operatorname{Var}^{\mathbb{Q}}\left(I_{2} \mid \mathcal{F}_{0}\right)+\sigma_{1}^{2} \operatorname{Var}^{\mathbb{Q}}\left(I_{3} \mid \mathcal{F}_{0}\right) \\
-2 \frac{\sigma_{2}^{2}}{\kappa^{2}} \operatorname{Cov}^{\mathbb{Q}}\left(I_{1}, I_{2} \mid \mathcal{F}_{0}\right)-2 \frac{\sigma_{1} \sigma_{2}}{\kappa} \operatorname{Cov}^{\mathbb{Q}}\left(I_{1}, I_{3} \mid \mathcal{F}_{0}\right) \\
+2 \frac{\sigma_{1} \sigma_{2}}{\kappa} \operatorname{Cov}^{\mathbb{Q}}\left(I_{2}, I_{3} \mid \mathcal{F}_{0}\right)
\end{array}
$$


Usando a isometria de Itô para o cálculo de variâncias e covariâncias, chegamos ao resultado:

$$
\begin{aligned}
\operatorname{Var}^{\mathbb{Q}}\left(\mathrm{X}_{\mathrm{t}} \mid \mathcal{F}_{0}\right)=( & \left.\sigma_{1}^{2}+\frac{\sigma_{2}^{2}}{\kappa^{2}}-\frac{2 \sigma_{1} \sigma_{2} \rho}{\kappa}\right) \mathrm{t}-2 \frac{\sigma_{2}^{2}}{\kappa^{3}}\left(1-\mathrm{e}^{-\kappa \mathrm{t}}\right) \\
& +2 \frac{\sigma_{1} \sigma_{2} \rho}{\kappa^{2}}\left(1-e^{-\kappa t}\right)+\frac{\sigma_{2}^{2}}{2 \kappa^{3}}\left(1-e^{-2 \kappa t}\right)
\end{aligned}
$$

Com os resultados acima, definimos a expressão para o preço futuro na data $t=0$ de um contrato que tem vencimento em $T$. Deve-se considerar este preço como sendo $F_{0, T}$. E observamos que $F_{0, T}=E^{\mathbb{Q}}\left(S_{T} \mid \mathcal{F}_{0}\right)$. Ou ainda:

$$
\ln F_{0, T}=\ln \left[E^{\mathbb{Q}}\left(S_{T} \mid \mathcal{F}_{0}\right)\right]
$$

Podemos descrever o valor esperado condicional de $S_{T}$ como:

$$
E^{\mathbb{Q}}\left(S_{T} \mid \mathcal{F}_{0}\right)=E^{\mathbb{Q}}\left(e^{\ln S_{T}} \mid \mathcal{F}_{0}\right)
$$

e desenvolvendo o segundo membro da equação, podemos observar que:

$$
\begin{aligned}
& E^{\mathbb{Q}}\left(S_{T} \mid \mathcal{F}_{0}\right)=\exp \left[E^{\mathbb{Q}}\left(\ln S_{T} \mid \mathcal{F}_{0}\right)+\frac{1}{2} \operatorname{Var}^{\mathbb{Q}}\left(\ln S_{T} \mid \mathcal{F}_{0}\right)\right] \\
& \ln \left[E^{\mathbb{Q}}\left(S_{T} \mid \mathcal{F}_{0}\right)\right]=E^{\mathbb{Q}}\left(\ln S_{T} \mid \mathcal{F}_{0}\right)+\frac{1}{2} \operatorname{Var}^{\mathbb{Q}}\left(\ln S_{T} \mid \mathcal{F}_{0}\right)
\end{aligned}
$$

Da Equação (Al), podemos descrever:

$$
E^{\mathbb{Q}}\left(\ln S_{t} \mid \mathcal{F}_{0}\right)=f_{t}+E^{\mathbb{Q}}\left(X_{t} \mid \mathcal{F}_{0}\right)
$$

Levando o resultado da Equação (A14) na Equação (A21), encontramos:

$$
E^{\mathbb{Q}}\left(\ln S_{t} \mid \mathcal{F}_{0}\right)=f_{t}+X_{0}+\left(r-\frac{1}{2} \sigma_{1}^{2}\right) t-\widehat{\alpha} t+\frac{\widehat{\alpha}-\delta_{0}}{\kappa}\left(1-e^{-\kappa t}\right)
$$

Levando as Equações (A17) e (A22) na Equação (A20) e considerando a data terminal $t=T$, temos o resultado: 


$$
\begin{array}{r}
\ln \left[E^{\mathbb{Q}}\left(S_{T} \mid \mathcal{F}_{0}\right)\right]=f_{T}+ \\
X_{0}+\left(r-\frac{1}{2} \sigma_{1}^{2}\right) T-\hat{\alpha} T+\frac{\hat{\alpha}-\delta_{0}}{\kappa}\left(1-e^{-\kappa T}\right) \\
+\left(\sigma_{1}^{2}+\frac{\sigma_{2}^{2}}{\kappa^{2}}-\frac{2 \sigma_{1} \sigma_{2} \rho}{\kappa}\right) T-2 \frac{\sigma_{2}^{2}}{\kappa^{3}}\left(1-e^{-\kappa T}\right) \\
+2 \frac{\sigma_{1} \sigma_{2} \rho}{\kappa^{2}}\left(1-e^{-\kappa T}\right)+\frac{\sigma_{2}^{2}}{2 \kappa^{3}}\left(1-e^{-2 \kappa T}\right)
\end{array}
$$

Usando a Equação (A18) e definindo por $A(T)$ a função dos termos que dependem do tempo e demais parâmetros, temos o resultado:

$$
\ln F_{0, T}=\ln S_{0}-\frac{\delta_{0}}{\kappa}\left(1-e^{-\kappa T}\right)+A(T)
$$

em que:

$$
\begin{array}{r}
A(T)=f_{T}+\left(r-\hat{\alpha}+\frac{1}{2} \frac{\sigma_{2}^{2}}{\kappa^{2}}-\frac{\sigma_{1} \sigma_{2} \rho}{\kappa}\right) T+\frac{1-e^{-\kappa T}}{\kappa^{2}}\left(\hat{\alpha} \kappa-\frac{\sigma^{2}}{\kappa}+\sigma_{1} \sigma_{2} \rho\right) \\
+\frac{\sigma_{2}^{2}}{4 \kappa^{3}}\left(1-e^{-2 \kappa T}\right)-f_{0}
\end{array}
$$

\section{Apêndice 2}

\section{Modelo na forma espaço de estado}

Neste apêndice, apresentamos o modelo na forma espaço de estado para a estimação via filtro de Kalman.

As variáveis de estado, preço à vista e retorno de conveniência não são diretamente observáveis, apenas o preço futuro é observável. A partir das Equações (2), (3) e (4), o modelo foi descrito sob a forma espaço de estado. Esta é a formulação usual para aplicação do filtro de Kalman e estimação dos hiperparâmetros, ou seja, os parâmetros do modelo não variam com o tempo.

Seja $N$ o número de contratos futuros e $\boldsymbol{y}_{t}$ o logaritmo dos preços futuros observados no mercado, conforme a Equação (4), podemos descrever que a equação de observação é:

$$
\boldsymbol{y}_{t}=\boldsymbol{d}_{t}+\boldsymbol{Z}_{t}\left[\boldsymbol{X}_{t}, \boldsymbol{\delta}_{t}\right]^{\top}+\boldsymbol{\epsilon}_{t}
$$


em que:

$$
\begin{aligned}
& \boldsymbol{y}_{t}=\ln \left[\boldsymbol{F}_{0, T_{i}}\right] \\
& \boldsymbol{d}_{t}=A\left(T_{i}\right) \\
& \boldsymbol{z}_{t}=\left[1,-\frac{1-e^{-\kappa T_{i}}}{\kappa}\right]
\end{aligned}
$$

$\boldsymbol{\epsilon}_{t}$ é um vetor $\mathrm{N} \times 1$, serialmente não correlacionado com média zero e matriz de covariância $\boldsymbol{H}_{t}$, ou seja: $E^{\mathbb{P}}\left(\boldsymbol{\epsilon}_{t}\right)=0$ e $\operatorname{Var}^{\mathbb{P}}\left(\boldsymbol{\epsilon}_{t}\right)=\boldsymbol{H}_{t}$.

As equações de transição do modelo são obtidas a partir das Equações (2) e (3) e são descritas como:

$$
\left[\boldsymbol{X}_{t}, \boldsymbol{\delta}_{t}\right]^{\top}=\boldsymbol{c}_{t}+\boldsymbol{Q}_{t}\left[\boldsymbol{X}_{t-1}, \boldsymbol{\delta}_{t-1}\right]^{\top}+\boldsymbol{\eta}_{t}
$$

em que:

$$
\begin{gathered}
\boldsymbol{c}_{t}=\left[\left(\mu-\frac{\sigma_{1}^{2}}{2}\right) \Delta t, \kappa \alpha \Delta t\right]^{\top} \\
\boldsymbol{Q}_{t}=\left[\begin{array}{cc}
1 & -\delta t \\
0 & 1-\kappa \Delta t
\end{array}\right]
\end{gathered}
$$

$\boldsymbol{\eta}_{t}$ é serialmente não correlacionado, com médias e variâncias sob a medida $\mathbb{P}$ dadas por:

$$
E^{\mathbb{P}}\left(\boldsymbol{\eta}_{t}\right)=0, \quad \operatorname{Var}^{\mathbb{P}}\left(\boldsymbol{\eta}_{t}\right)=\left[\begin{array}{cc}
\sigma_{1}^{2} \Delta_{t} & \rho \sigma_{1} \sigma_{2} \Delta t \\
\rho \sigma_{1} \sigma_{2} \Delta t & \sigma_{1}^{2} \Delta t
\end{array}\right]
$$




\section{Referências}

Aiube, F. A. L., T. K. N. Baidya and E. A. H. Tito. “Analysis of Commodity Prices with the Particle Filter". Energy Economics 30, (2008): 597-605.

Alquist, R., L. Kilian and R.J. Vigfusson. "Forecasting the Price of Oil". In the G. Elliott and A. Timmermann (eds.), Handbook of Economic Forecasting 2, Amsterdam, 2013.

Babbs, S. H. and K. B. Nowman. "Kalman Filtering of Generalized Vasicek Term Structure Models." Journal of Financial and Quantitative Analysis 34, no. 1 (1999): 115-130.

Baumeister, C., and L. Kilian "Forty Years of Oil Price Fluctuations: Why the Price of Oil May Still Surprise Us". Journal of Economic Perspectives 30, (2016): 139-160.

Bessembinder, H., J. F. Coughenour, M. Smoller, and P. J. Seguin. "Mean Reversion in Equilibrium Asset Prices: Evidence from the Futures Term Structure.” Journal of Finance 50, no. 1 (1995): 361-375.

Brennan, M., and E. S. Schwartz. "Evaluating Natural Resource Investments.” The Journal of Business 58, no. 2, (1985): 135-157.

Campbell, J. Y., A. Lo, and, A. C. MacKinley. The econometrics of financial markets. Princeton University Press, 1997.

Cortazar, G. and L. Naranjo, “An N-factor Gaussian model of oil futures prices.” Journal Future Markets 26, (2006): 243-268.

Durbin, J. and S. J. Koopman. Time Series Analysis by State Space Methods. Oxford University Press, 2002.

Geman, H. Commodities and Commodity Derivatives: Modeling and Pricing for Agriculturals, Metals and Energy. John Wiley \& Sons, Ltd, 2005.

Geman, H. and V. Nguyen, "Soybean inventory and forward curve dynamics." Management Science 51, no. 7, (2005):1076-1091.

Gibson, R. and E. S. Schwartz. "Stochastic Convenience Yield and the Pricing of Oil Contingent Claims." Journal of Finance 45, no. 3, (1990):959-976.

Hamilton, J. D. Time Series Analysis. Princeton: Princeton University Press, 1994

Hamilton, J. D. and J. C. Wu. "Risk premia in crude oil futures prices." Journal of International Money and Finance 42, (2014) 9-37.

Harvey, A. Forecasting, Structural Time Series Models and the Kalman Filter. Cambridge: Cambridge University Press, 1989.

Hikspoors, S. and S. Jaimungal, "Energy spot price models and spread options pricing." International Journal of Theoretical and Applied Finance 10, no. 7, (2007): 1111-1135.

Javaheri, A., D. Lautier, and A. Galli. "Filtering in Finance”. Wilmott Magazine 3, (2003): 67-83.

Kaldor, N. "Speculation and Economic Stability." The Review of Economic Studies 7, (1939): 1-27, http://dx.doi. org/10.2307/2967593.

Kilian, L. "Oil price shocks: Causes and consequences." Annual Review of Resource Economics 6, no. 1, (2014): 133-154.

Lucia, J. J., and E. S. Schwartz. "Electricity prices and power derivatives: Evidence from the Nordic Power Exchange." Review of Derivatives Research, (2001): 5-50.

Manoliu, M., and S. Tompaidis. "Energy futures prices: term structure models with Kalman filter estimation." Applied Mathematical Finance, 9, no. 1, (2002): 21-43.

Ministério da Indústria, Comércio Exterior e Serviços. "Comex Vis: Principais Produtos Exportados.” Acessado em março de 2018. http://www.mdic.gov.br/comercio-exterior/estatisticas-de-comercio-exterior/comex-vis/frame-ppe Pilipovic, D. Energy Risk: Valuing And Managing Energy Derivatives. New York: McGraw-Hill, 1998.

Pindyck, R. S. “The Long-Run Evolutions of Energy Prices.” The Energy Journal 20, no. 2, (1999): 1-27. 
Postali, F. A. and Picchetti, P. "Geometric Brownian Motion and structural breaks in oil prices: A quantitative analysis." Energy Economics 28, no. 4, (2006): 506-522.

Prokopczuk K, M. and Y. Wu. "The Determinants of Convenience Yields." University of Reading and Zeppelin University Working Paper (2013).

Schwartz, E. S. "The Stochastic Behavior of Commodity Prices: Implications for Valuation and Hedging." Journal of Finance 52, no. 3, (1997): 923-973.

Schwartz, E. S. and, J. E. Smith. "Short-Term Variations and Long-Term Dynamics in Commodity Prices." Management Science 46, no. 7, (2000): 893-911.

Shafiee, S., and E. Topal. "A long-term view of worldwide fossil fuel prices". Applied Energy, no. 87, (2010): 988-1000.

Sørensen, C. "Modeling seasonality in agricultural commodity futures." Journal of Futures Markets 22, no. 5, (2002): 93-426.

Villaplana, P. “Pricing Power Derivatives: A Two-Factor Jump-Diffusion Approach.” Working paper, Universidad Carlos III de Madrid, 2003. 